Glasgow Caledonian

University

University for the Common Good

\title{
What is the impact of a country-wide scale-up in antiviral therapy on the characteristics and sustained viral response rates of patients treated for hepatitis $\mathbf{C}$ ?
}

McDonald, Scott A.; Innes, Hamish A.; Hayes, Peter C.; Dillon, John F.; Mills, Peter R.; Goldberg, David J.; Barclay, Stephen; Allen, Sam; Fox, Ray; Fraser, Andrew; Kennedy, Nicholas; Bhattacharyya, Diptendu; Hutchinson, Sharon J.

Published in:

Journal of Hepatology

DOI:

10.1016/j.jhep.2014.08.046

Publication date:

2015

Document Version

Author accepted manuscript

Link to publication in ResearchOnline

Citation for published version (Harvard):

McDonald, SA, Innes, HA, Hayes, PC, Dillon, JF, Mills, PR, Goldberg, DJ, Barclay, S, Allen, S, Fox, R, Fraser, A, Kennedy, N, Bhattacharyya, D \& Hutchinson, SJ 2015, 'What is the impact of a country-wide scale-up in antiviral therapy on the characteristics and sustained viral response rates of patients treated for hepatitis C?', Journal of Hepatology, vol. 62 , no. 2, pp. 262-268. https://doi.org/10.1016/j.jhep.2014.08.046

\section{General rights}

Copyright and moral rights for the publications made accessible in the public portal are retained by the authors and/or other copyright owners and it is a condition of accessing publications that users recognise and abide by the legal requirements associated with these rights.

Take down policy

If you believe that this document breaches copyright please view our takedown policy at https://edshare.gcu.ac.uk/id/eprint/5179 for details of how to contact us. 
What is the impact of a country-wide scale-up in antiviral therapy on the characteristics and sustained viral response rates of patients treated for hepatitis $C$ ?

Scott A McDonald ${ }^{1,2}$, Hamish A Innes ${ }^{1,2}$, Peter C Hayes ${ }^{3}$, John F Dillon ${ }^{4}$, Peter R Mills ${ }^{5}$, David J Goldberg, ${ }^{2,1}$, Stephen Barclay ${ }^{6}$, Sam Allen7, Ray Fox ${ }^{5}$, Andrew Fraser ${ }^{8}$, Nicholas Kennedy ${ }^{9}$, Diptendu Bhattacharyya ${ }^{10}$, Sharon J Hutchinson ${ }^{1,2}$

${ }^{1}$ School of Health and Life Sciences, Glasgow Caledonian University, Cowcaddens Road, Glasgow G4 0BA, Scotland, UK

${ }^{2}$ Health Protection Scotland, Meridian Court, 5 Cadogan Street, Glasgow G2 6QE, Scotland, UK

${ }^{3}$ Edinburgh Royal Infirmary, Edinburgh Scotland, UK

${ }^{4}$ Ninewells Hospital and Medical School, Dundee, Scotland, UK

${ }^{5}$ Gartnavel General Hospital, Glasgow, Scotland, UK

${ }^{6}$ Glasgow Royal Infirmary, Glasgow, Scotland, UK

${ }^{7}$ Crosshouse Hospital, Kilmarnock, Scotland, UK

${ }^{8}$ Aberdeen Royal Infirmary, Aberdeen, Scotland, UK

${ }^{9}$ Monklands Hospital, Airdrie, Scotland, UK

${ }^{10}$ Kirkcaldy Hospital, Kirkcaldy, UK

\section{Corresponding author and address where work was done}

Scott A. McDonald

Health Protection Scotland

Meridian Court, 5 Cadogan Street,

Glasgow G2 6QE, Scotland, UK

Email: smcdonald4@nhs.net

Tel: +441413001106

Fax: +44 1413001170

Word count: 4773 ; abstract: 250; number of figures: 3 ; number of tables: 2

List of abbreviations: $\mathrm{HCV}=$ hepatitis $\mathrm{C}$ virus; $\mathrm{PWID}=$ people who inject drugs; $\mathrm{SVR}=$ sustained viral response; ESLD = end-stage liver disease

Conflicts of interest: none declared 
ABSTRACT

\section{Background and aims}

The global burden associated with hepatitis $\mathrm{C}$ virus $(\mathrm{HCV})$ infection has prompted a scale-up of antiviral therapy. Hitherto, no data existed on the impact of scaling-up on the characteristics of treated populations or on sustained viral response (SVR) rates. We assessed the country-wide scale-up of antiviral therapy in Scotland, a country which nationally monitors uptake of and response to $\mathrm{HCV}$ treatment

\section{Methods}

Data for patients initiated on combined pegylated interferon and ribavirin therapy at 13 specialist HCV clinics in 2001-2010 were extracted from the Scottish HCV Clinical Database ( $n=3895)$. Patient characteristics included age, genotype, PWID (people who inject drugs) status, prison referral, and diagnosed cirrhosis. Temporal trends in covariates and adjusted effects on SVR were examined via mixed-effects regression.

\section{Results}

The number of patients starting treatment increased from 237 in 2001-2002 to 1560 in 20092010, with an increasing trend in SVR from $44 \%$ to 57\% over this period. For a given clinic, between 2001/2 and 2010 there was a decrease in the odds of those treated being diagnosed cirrhotic (odds ratio[OR]=0.84 per year), and increasing temporal trends for those treated being PWID (OR=1.08) and prison referral ( $\mathrm{OR}=1.06)$. Adjusting for covariates, the proportion of a given clinic's patients achieving SVR was positively associated with the percentage PWID (OR=1.01 per percent increase; 95\% confidence interval [CI]: 1.00-1.02) and genotype 2/3 (OR=1.03; 95\% CI: 1.02-1.04).

\section{Conclusions}


Despite changes in patient characteristics, a country-wide scale-up of antiviral therapy did not compromise SVR rates. Results are highly relevant to countries planning on scaling-up treatment, given the forthcoming availability of new interferon-free therapies.

KEYWORDS: hepatitis C virus, antiviral therapy, pegylated interferon, sustained viral response 


\section{INTRODUCTION}

The global public health burden associated with hepatitis $\mathrm{C}$ virus (HCV) infection is growing,[1] with an estimated >185 million HCV antibody-positive individuals in 2005, relating to a global prevalence of $2.8 \%$. [2] At the national level, disease modelling exercises [3-7]) suggest that HCV-related disease burden will continue to increase with current low rates of antiviral treatment. These models illustrate that the annual number of HCV patients initiated on treatment would need to be radically increased to counteract the projected rise in disease burden.

In Scotland, a country with approximately 39,000 people currently estimated to be chronically infected with HCV (0.8\% of the general population),[8] the annual numbers of patients initiated on antiviral therapy has approximately doubled between 2007/2008 ( $n=$ 468) and 2010/2011 ( $n=1049)$ as a result of the Scottish Government's HCV Action Plan[9] and significant investment. $[8,10]$ This Plan aimed to ensure those infected receive optimal treatment, care and support, through nationally agreed guidelines, standards and targets and accountable national and local multi-disciplinary networks responsible for the planning, development and delivery of services. A considerable emphasis was placed on co-ordination and monitoring to ensure progress was made. A HCV clinical database was thus established in almost all specialist centres delivering $\mathrm{HCV}$ treatment - a data-monitoring initiative unique to Scotland - and provided an opportunity to explore the impact of this scaling-up in the delivery of antiviral therapy, with respect to both the characteristics and sustained viral response (SVR) rates of treated patients. Data at the international level is non-existent in this respect, despite recent increases in the number of patients treated for HCV across parts of Europe, Asia, and the Middle East.[11] Moreover, it was unknown if scaling-up might have an associated reduction in SVR as a consequence of, for instance, specialist services being unable to cope with new demand and the administration of therapy to less-compliant 
individuals. This investigation will be highly relevant and provide vital evidence for other countries either planning or in the process of scaling-up HCV treatment.

\section{METHODS}

\section{Data source}

We extracted the clinical treatment data for all 17 specialist clinics delivering HCV treatment in Scotland, over the 10-year period from the start of 2001 to the end of 2010, from the Scottish Hepatitis C Clinical Database held at Health Protection Scotland.[12] The patient population was restricted to those initiated on combined pegylated interferon and ribavirin (pegI\&R) antiviral therapy, because we wished to distinguish any changes in SVR from improvements in antiviral therapy available. A total of 3,999 patients were initiated on at least one pegI\&R treatment course within the study period.

\section{Design and variables}

Thirteen specialist clinics providing outpatient HCV treatment were included in analysis: Crosshouse Hospital, Dumfries \& Galloway Royal Infirmary, Ninewells Hospital, Aberdeen Royal Infirmary, Gartnavel General Hospital (Infectious Diseases), Gartnavel General Hospital (Gastroenterology), Glasgow Royal Infirmary, Kirkcaldy Hospital, Monklands Hospital, Raigmore Hospital, Royal Infirmary Edinburgh, Southern General Hospital, and Stirling Royal Infirmary (total $N=3895$ ), with four clinics (Borders General Hospital, Hairmyres Hospital, Inverclyde Royal Hospital, Royal Alexandra Hospital) excluded, as fewer than 50 patients in these clinics were initiated on treatment over the study period. Data points corresponded to clinic/yearof-treatment-initiation combinations. Data for the first two years of the study period (2001 and 2002) were aggregated, because there were few pegI\&R treatment initiations for the majority of the clinics at that time. The primary outcome variable was sustained viral response (SVR), which was defined as a documented negative PCR test at least 6 months 
following termination of treatment. All patients without a documented PCR test at least 6 months following treatment termination were classified as non-SVR. Covariates of interest included male sex, age at treatment initiation, risk group (known PWID, known non-PWID, not known), referral from prison setting (yes / no), diagnosed cirrhosis (yes / no), maximum sustained weekly alcohol consumption ( $\geq 50$ units, $<50$ units/week) (self-reported; set to $<50$ if unknown/not reported), and HCV genotype (2, 3 / all other genotypes). Diagnosis of cirrhosis was based on one or more of the following criteria: (i) clinical examination; (ii) radiology (i.e., ultrasound, FibroScan, computed tomography, or magnetic resonance imaging procedure); (iii) liver biopsy.[12] All variables were treated as cross-sectional variables, meaning that SVR was converted to a proportion, and all covariates except age were converted to percentages within each clinic-year combination; mean age was calculated within each clinic-year.

\section{Statistical analyses}

Analysis (a) investigated temporal trends in patients characteristics over the study period. Mixed-effects linear regression models were fitted separately for each covariate, with year as the single fixed effect (with 2001/2002 coded as 2001.5) and clinic specified as a random intercept, given the non-independence of observations within clinics. For analyses with a proportion as the response variable (i.e., all covariates except age), the logit link function was specified; for these models, $\beta$ coefficients are interpreted as log odds ratios, so are instead reported as $\exp (\beta)$, the odds ratio. PWID was defined as the proportion of PWID risk among patients with known risk only (i.e. patients with the status not known were excluded).

Analysis (b) describes temporal trends in SVR over the study period, by fitting a mixedeffects linear regression model with year as the single fixed effect and clinic as a random intercept. A comparable analysis was also conducted on the study population excluding 
patients with diagnosed cirrhosis.

In analysis (c), we fitted time-series cross-sectional (TSCS) regression models appropriate for our data; these models can specify grouping variables as fixed or random effects. We attempted to isolate any unique effects on SVR that were associated with changing patient characteristics due to broadening of the eligible treatment patient population (e.g., the percentage of PWID patients, the percentage of referrals from prison settings) over the study period, by adjusting for other covariates. A Hausman test was conducted to assess the appropriateness of specifying clinic as a random effect rather than a fixed effect, and the need to include year as an additional fixed effect was assessed using a Lagrange multiplier test. In this type of regression model, a single regression coefficient fitted for a given covariate (e.g., PWID) aggregates both between-clinic and within-clinic effects (i.e., time-invariant and timevarying components of the covariate effect).[13] Therefore, a test for equality of these parameters was conducted before final model specification, $[13,14]$ and if necessary, separate covariates were fitted in the final model instead: the cluster mean $\left(x_{j}\right)$, representing the between-cluster effect, and the deviation from the cluster mean $\left(x_{j}-x_{i j}\right)$, representing the within-clinic, or temporal, effect.

To compare covariate effects obtained with analysis of the TSCS view of the data, we also conducted an individual-level analysis by fitting a multifactorial random-effects binomial regression model to the same dataset. Risk group was necessarily fitted as a categorical variable (known PWID, known non-PWID, not known). Note that the individual-level approach does not allow the main research question to be addressed (i.e., investigation of the associations of changing clinic patient characteristics over time with [changes in] SVR rates over time). 
All regression models were fitted using the $\operatorname{lme} 4[15]$ and plm[16] packages for the $R$ statistical computing environment (version 3.0.0).[17]

\section{RESULTS}

Figure 1 illustrates the growth of the treated population over the study period, showing both the cumulative numbers of patients initiated on pegI\&R treatment and the cumulative number of SVR achieved, in 17 Scottish specialist clinics over the period 2001-2010. Table 1 summarises the characteristics of our study population according to calendar year period of initiation on antiviral therapy. There was an increase in the overall number of patients starting treatment over time (see also Fig. 1), from 237 in 2001-2002 to 1560 in 2009-2010. The number of treated patients achieving an SVR also increased from 104 (44\%) in 2001-2002 to 882 (57\%) in 2009-2010; excluding those patients with diagnosed cirrhosis, SVR increased from $51 \%$ in $2001-2002$ to $59 \%$ in $2009-2010$. Over the same period, the proportion of treated patients with known risk group that were current/former PWID increased from 112/212 (53\%) to 1023/1351 (76\%), and the proportion with diagnosed cirrhosis decreased from 30\% to $11 \%$. The average age at treatment initiation was 41 years (SD $=9.5$ years), which was stable across time.

Trends over time (analysis (a)) for the principal covariates are displayed in Figure 2, in which data are grouped according to clinic and calendar year of treatment initiation (which may differ from calendar year of SVR if therapy was begun later in the year). Between 2001/2 and 2010, there was a statistically significant decreasing trend observed for the within-clinic proportion of treated patients with diagnosed cirrhosis $(\mathrm{OR}=0.84, p<0.0001$; i.e. there was a $16 \%$ decrease in the odds of a treated patient having diagnosed cirrhosis). Significant 
increasing temporal trends were found for the within-clinic proportion of treated patients with PWID risk $(\mathrm{OR}=1.08, p=0.026)$ and prison referral $(\mathrm{OR}=1.06, p=0.002)$. In the last year of the study period, an across-clinic mean of $57 \%, 11 \%, 77 \%$ and $6.7 \%$ treated patients were HCV genotype 2 or 3, had diagnosed cirrhosis, were current/former PWID, or were referred from a prison setting, respectively.

The SVR temporal trend (analysis (b)) for individual clinics, and the trend of the mean over all clinics, is displayed in Fig. 3. At the clinic level, there was a non-significant overall increasing trend in the proportion of treated patients achieving SVR over the study period (odds ratio $[\mathrm{OR}]$ per year $=1.03, p=0.33$; in other words, a $3 \%$ increase in the odds of achieving SVR per year), from an across-clinic mean of 44\% in 2001/2002 to 56\% in 2010. Stratified analyses conducted according to genotype (i.e., separately for genotype $2 / 3$ and for other genotypes) indicated similar SVR temporal trends (Fig. 3). A supplementary analysis (Appendix, Table A2 and Fig. A2) conducted on only those patients without diagnosed cirrhosis $(n=3297)$ also did not indicate any trend in the proportion achieving SVR, from across-clinic mean of $51 \%$ in $2001 / 2$ to $58 \%$ in 2010 (OR $=0.97, p=0.38)$. Note that the lines indicating across-clinic means in these SVR plots do not show variability, and thus only serve to visually indicate temporal trends.

The covariates of interest are all plausibly associated with SVR, but apparent crude associations between a temporal trend in SVR and a trend in a particular covariate may be confounded by other, related variables. Therefore, we also estimated the associations between within-clinic proportions achieving SVR and within-clinic patient characteristics after adjusting for other covariates (analysis (c)). Male sex and weekly alcohol consumption were excluded from the adjusted model, as no crude association was observed with SVR. Based on the Hausman test result, clinic was specified as a random effect. The final fitted mixed-effects 
regression model is presented in Table 2 and the regression equation is below:

$\operatorname{logit}\left(y_{i j}\right)=\beta_{0 j}+\beta_{1} x_{1 i j}+\beta_{2} x_{2 i j}+\beta_{3} x_{3 i j}+\beta_{4} x_{4 i j}+\beta_{5} x_{5 i j}+u_{j}+\varepsilon_{i j}$

where $\beta_{1^{\prime}} \beta_{2^{\prime}} \beta_{3^{\prime}} \beta_{4}$, and $\beta_{5}$ are the coefficients for age, cirrhosis, genotype $2 / 3, P W I D$, and prison referral, respectively, and $u_{j} \sim N\left(0, \sigma^{2}\right)$.

Adjusting for the other covariates, there was a significant 1.027-fold increase in the odds of SVR (95\% CI: 1.016-1.037) with every percentage increase in HCV genotype 2/3 patients treated over time (Table 2). For a given clinic, there was a statistically significant 1.012-fold increased odds of SVR with every percentage increase in PWID treated over time.

In individual-level analyses (Appendix, Table A1), there was a statistically significant decreased odds of SVR for a patient associated with each 1-year increase in their age $(\mathrm{OR}=$ 0.977 ; i.e. a $2.3 \%$ decrease in the odds of SVR per year of age), and a significant decreased odds of SVR with diagnosed cirrhosis $(\mathrm{OR}=0.26$, which is equivalent to $\mathrm{OR}=0.987$ per percentage decrease, to allow easy comparison with the TSCS results, in which cirrhosis is a proportion rather than a binary variable). The individual-level analysis also indicated decreased odds of SVR for PWID compared with non-PWID risk $(\mathrm{OR}=0.82)$, and increased odds of SVR for genotype 2/3 compared with other genotypes $(\mathrm{OR}=2.77)$.

\section{DISCUSSION}

The current study represents the first evidence investigating temporal trends in response to HCV antiviral therapy. Despite a country-wide scale-up of chronic HCV-infected patients initiated on pegI\&R antiviral therapy over the study period - from 237 patients in 2001-2002 to 1560 in 2009-2010 - and changes in patient characteristics over the same period - SVR 
rates were not compromised. Temporal trends in several patient characteristics known to be associated with SVR rates[12] were found; namely, within clinics there were increased odds of treated patients with PWID status and referred from prison settings, and decreased odds of treated patients with diagnosed cirrhosis, but there was no evidence that the proportion of patients achieving an SVR was affected. There was also no evidence that the greater proportion of diagnosed cirrhotic patients treated in the earlier part of the study period concealed a downward trend in SVR rates, as excluding these patients from analysis did not change the results.

Our main finding authenticates the assumption - of SVR rates being maintained, despite the broadening of the treated patient base - employed in numerous modelling studies (recently those reported in $[18,19])$ predicting the impact of scaling-up therapy.

For a given clinic, a higher proportion of SVR was associated with genotype $2 / 3 \mathrm{HCV}$ compared with all other genotypes (adjusting for other covariates), which is consistent with previous studies.[12, 20-22] Both the unadjusted and adjusted TSCS analysis indicated a positive association between SVR and the proportion of patients who were current or former PWID, which was unexpected. This cannot be interpreted as a causal association (e.g., more PWID initiated on treatment gives rise to a greater SVR rate), because a single regression coefficient in the TSCS analysis aggregates time-invariant and time-varying components of the covariate effect. That is, a greater proportion of PWID may be treated within clinics that have better overall SVR rates than within clinics with lower rates.[14] Because the individuallevel analysis indicated that PWID risk was associated with a significantly decreased odds of achieving an SVR (OR=0.82, where non-PWID risk was the reference group), PWID is likely confounded with age and being diagnosed with cirrhosis in the TSCS analysis. Clinics that treat younger patients and/or a smaller proportion of patients with diagnosed cirrhosis will 
tend to have better clinic-level SVR rates, all else being equal. Because PWID tend to be younger and have less progressed liver disease, including PWID in the adjusted model tends to conceal the effects of age and diagnosed cirrhosis, indicated by the change in regression coefficient from $<1$ (unadjusted) to $>1$ (adjusted).

In the context of a continuing rising trend in annual number of $\mathrm{HCV}$ infected persons developing and dying from end-stage liver disease (ESLD) in Scotland,[8] the finding of a significant decrease in the odds of those treated being diagnosed with cirrhosis over time is concerning. To curb the ongoing rise in ESLD in the short term, treatment of those with more advanced disease is necessary. The absolute number of diagnosed cirrhosis patients of course still increased over time (doubling between 2001-2 and 2009-10); but not to the same extent as the overall number treated (with an almost seven-fold rise between 2001-2 and 2009-10) and the number of PWID treated (with a nine-fold rise between 2001-2 and 2009-10). The expansion of treatment among PWID relates to the Scottish Government's HCV Action Plan and initiatives to increase diagnosis and treatment particularly among this group, with the introduction of dried blood spot testing in specialist addiction services.[23] As liver biopsy was one of the diagnostic criteria for cirrhosis, the declining odds of treated patients being diagnosed cirrhotic could have been exaggerated due to the change in biopsy practice - the biopsy rate dropped from $14 \%$ in $2000-2004$ to $3 \%$ in $2005-2010$ - and so the prevalence of cirrhosis among our patients may have been under-estimated to a greater extent in recent years. However, from another study which examined FibroScan data, we estimated that 15\% of patients treated for HCV in Scotland during 2009-2013 were cirrhotic [H. Innes, pers. comm.], which is only slightly higher than that observed here for patients treated during 2009-2010 (10\%). Thus, our findings still suggest that greater effort is needed to identify and treat those with more advanced disease. 
We restricted the dataset to treatment initiations on pegI\&R; otherwise, we could not distinguish a possible rising trend in SVR rates from improvements in antiviral therapy during the same period. However, the proportion achieving a SVR was quite stable over the study period. Another limitation is the paucity of data in the earliest years of the study period. In 2001-2, there was an average of 14 patients per clinic initiated on antiviral therapy; therefore the clinic-specific covariate estimates in the early part of the study period will not be as stable as estimates for later years.

Our intention-to-treat analysis, while appropriate for the research questions, also means that SVR proportions are lower than if we had excluded patients without PCR test results following the end of treatment $(n=134 ; 3.4 \%)$ or persons otherwise lost to follow-up from the denominator. This would slightly restrict the variability in SVR across clinics and over time, weakening the strength of possible associations with the covariates of interest.

Finally, because active injecting status is not recorded in the Scottish Hepatitis C Clinical database, we were unable to determine whether the rising trend observed in the proportion of treatment patients who are PWID (i.e., those either currently or formerly injecting drugs) also applies to those who were actively injecting drugs. It may be that increasing numbers of relatively stable patients with a past history of injecting drug use and with mild disease were initiated on treatment, possibly as a consequence of the Scottish Government's HCV Action Plan.[9] If younger PWID (i.e.,. $<35$ years of age) are taken as a proxy for active injectors, then a rising trend in this group is still apparent (data not shown). The role of antiviral therapy in reducing transmission among PWID has been previously highlighted in modelling studies,[24] and there is accumulating evidence that the scale-up of interventions among those who actively inject in Scotland - principally injecting equipment provision and opiate substitute therapy, but potentially also antiviral therapy - has lead to a reduction in 
the incidence of HCV infection.[25-27]

Antiviral treatment uptake remains low internationally; with $<5 \%$ of chronically infected patients treated per year across most of Europe,[28] Australia,[29] and USA.[30] There are however promising new interferon-free therapies forthcoming: so called direct-acting antivirals (DAA), which should allow much higher SVR rates to be achieved, even among those with cirrhosis.[31, 32] The availability of these new effective treatments will mean that countries will be looking at scaling-up therapy delivery at the national level. In Scotland, the scale-up in treatment uptake was achieved[10] by a combination of major investment (43 million pounds during 2008/09 to 2010/11), the development of national guidelines and targets for clinical services, affiliation of NHS boards to multi-disciplinary managed HCV care networks and appointment of HCV executive and clinical leads accountable for local performance, among other initiatives such as awareness-raising campaigns and the national procurement of antiviral drugs.[23]

Although published data on the impact of treatment scale-up on SVR rates in other countries are scarce, success (in terms of treatment uptake) is notable for France, who have had a National Plan for Hepatitis B and C in place since 1999.[33] France achieved a treatment rate of $16 \%$ in 2005 , and a reported 10,000 patients were initiated on antiviral therapy in 2011.[34] Common to both National strategies in France and Scotland has been a robust evidence base and reliable epidemiological data to drive policy, with quantifiable goals providing important motivation for success.[35]

In summary, the comprehensiveness of Scotland's clinical treatment database has provided a unique opportunity to investigate the impact on SVR rates of broadening treatment to those 
patient groups who are often ineligible for clinical trials (e.g., PWID, incarcerated individuals), but nevertheless account for the majority of the chronically infected population. SVR rates did not appear to be compromised, despite the broadening of the eligible patient base associated with the implementation of the HCV Action Plan and other initiatives. Monitoring the characteristics of treated patients will be vitally important over the coming years to ensure that the patients who receive treatment are those who are most in need. The present results are highly relevant to countries planning on scaling-up treatment, given the forthcoming availability of new interferon-free therapies.

\section{ACKNOWLEDGEMENTS}

We thank the Hepatitis C clinical Database Monitoring committee, clinical data entry staff and the following virologists for their support with the HCV diagnosis database: Dr Sheila Burns (East of Scotland Specialist Virology Centre, Royal Infirmary of Edinburgh, Edinburgh), Dr Sheila Cameron (West of Scotland Specialist Virology Centre, Gartnavel General Hospital, Glasgow), Dr Paul McIntyre (Department of Medical Microbiology, Ninewells Hospital and Medical School, Dundee) and Dr Pamela Molyneaux (Department of Medical Microbiology, University Medical School, Foresterhill, Aberdeen). 


\section{REFERENCES}

[1] Global Burden Of Hepatitis C Working Group. Global burden of disease (GBD) for hepatitis C. J Clin Pharmacol 2004; 44:20-29.

[2] Mohd Hanafiah K, Groeger J, Flaxman AD, Wiersma ST. Global epidemiology of hepatitis $\mathrm{C}$ virus infection: new estimates of age-specific antibody to $\mathrm{HCV}$ seroprevalence. Hepatology $2013 ; 57: 1333-1342$.

[3] Law M, Dore G, Bath N, Thompson S, Crofts N, Dolan K. Modelling hepatitis C virus incidence; prevalence and long-term sequelae in Australia; 2001. Int J Epidemiol 2003; 32:71724.

[4] Hutchinson S, Bird S, Goldberg D. Modeling the current and future disease burden of hepatitis C among injection drug users in Scotland. Hepatology 2005; 42:711-23.

[5] Sweeting M J, De Angelis D, Brant LJ, Harris HE, Mann AG, Ramsay ME. The burden of hepatitis C in England. J Viral Hepat 2007; 14:570-576.

[6] Davis G, Albright J, Cook S, Rosenberg D. Projecting future complications of chronic hepatitis C in the United States. Liver Transpl 2003; 9:331-8.

[7] Deuffic-Burban S, Mathurin P, Valleron AJ. Modelling the past, current and future HCV burden in France: detailed analysis and perspectives. Stat Methods Med Res 2009; 18:233-252. [8] Health Protection Agency. Hepatitis C in UK 2012. London: Health Protection Agency Centre for Infections, 2012.

[9] Scottish Government Health Department. Hepatitis C Action Plan for Scotland: Phase II: May 2008 - March 2011. Edinburgh: Scottish Government, 2008.

[10] McDonald S A, Hutchinson SJ, Innes HA, Allen S, Bramley P, Bhattacharyya D et al. Attendance at specialist hepatitis clinics and initiation of antiviral treatment among persons chronically infected with hepatitis C: examining the early impact of Scotland's Hepatitis C Action Plan. J Viral Hepat 2014; 21:366-376.

[11] Razavi H, Estes C, Pasini K, Levitch A. The Number of Treated HCV Infected Patients in Select Countries/Regions in 2004-2010. Poster presented at the 63rd Annual Meeting of the American Association for the Study of Liver Diseases November 9-13. Boston, MA, 2012. [12] Innes H A, Hutchinson SJ, Allen S, Bhattacharyya D, Bramley P, Carman B et al. Ranking predictors of a sustained viral response for patients with chronic hepatitis $C$ treated with 
pegylated interferon and ribavirin in Scotland. Eur J Gastroenterol Hepatol 2012; 24:646-655.

[13] Bartels B. Beyond "fixed versus random effects": a framework for improving substantive and statistical analysis of panel, time-series cross-sectional, and multilevel data. The Society for Political Methodology 2008:1-43.

[14] Leyland A H. No quick fix: understanding the difference between fixed and random effect models. J Epidemiol Community Health 2010; 64:1027-1028.

[15] Bates D, Maechler M. lme4: Linear mixed-effects models using S4 classes. R package version 0.999375-32 2013.

[16] Croissant Y, Milo G. Panel Data Econometrics in R: The plm Package. Journal of Statistical Software 2008; 27(2).

[17] R Development Core Team. R: A language and environment for statistical computing. Vienna; Austria: R Foundation for Statistical Computing, 2014.

[18] Razavi H, Waked I, Sarrazin C, Myers RP, Idilman R, Calinas F et al. The present and future disease burden of hepatitis $\mathrm{C}$ virus $(\mathrm{HCV})$ infection with today's treatment paradigm. J Viral Hepat 2014; 21 Suppl 1:34-59.

[19] Wedemeyer H, Duberg AS, Buti M, Rosenberg WM, Frankova S, Esmat G et al. Strategies to manage hepatitis C virus (HCV) disease burden. J Viral Hepat 2014; 21 Suppl 1:60-89.

[20] Fried M W, Shiffman ML, Reddy KR, Smith C, Marinos G, Goncales FL,Jr et al. Peginterferon alfa-2a plus ribavirin for chronic hepatitis C virus infection. N Engl J Med 2002; 347:975-982.

[21] Hadziyannis S J, Sette H,Jr, Morgan TR, Balan V, Diago M, Marcellin P et al. Peginterferon-alpha2a and ribavirin combination therapy in chronic hepatitis C: a randomized study of treatment duration and ribavirin dose. Ann Intern Med 2004; 140:346355.

[22] Manns M P, McHutchison JG, Gordon SC, Rustgi VK, Shiffman M, Reindollar R et al. Peginterferon alfa- $2 b$ plus ribavirin compared with interferon alfa- $2 b$ plus ribavirin for initial treatment of chronic hepatitis C: a randomised trial. Lancet 2001; 358:958-965.

[23] Hutchinson S, Goldberg D, Brown G, Rowan N, Dillon J, Taylor A et al. Hepatitis C strategy in Scotland. Viral hepatitis in practice 2012; 4(2). 
[24] Martin N K, Vickerman P, Foster GR, Hutchinson SJ, Goldberg DJ, Hickman M. Can antiviral therapy for hepatitis $\mathrm{C}$ reduce the prevalence of $\mathrm{HCV}$ among injecting drug user populations? A modeling analysis of its prevention utility. J Hepatol 2011; 54:1137-1144. [25] Palmateer N E, Taylor A, Goldberg DJ, Munro A, Aitken C, Shepherd SJ et al. Rapid decline in $\mathrm{HCV}$ incidence among people who inject drugs associated with national scale-up in coverage of sterile injecting equipment and opiate substitution therapy. PloS One. Submitted.

[26] Turner K M, Hutchinson S, Vickerman P, Hope V, Craine N, Palmateer N et al. The impact of needle and syringe provision and opiate substitution therapy on the incidence of hepatitis C virus in injecting drug users: pooling of UK evidence. Addiction 2011; 106:19781988.

[27] Allen E J, Palmateer NE, Hutchinson SJ, Cameron S, Goldberg DJ, Taylor A. Association between harm reduction intervention uptake and recent hepatitis $C$ infection among people who inject drugs attending sites that provide sterile injecting equipment in Scotland. Int J Drug Policy 2012; 23:346-352.

[28] Razavi H, Estes C, Pasini K, Gower E, Hindman S. Treatment rate in select European countries in 2004-2010. Conference of the European Association for the Study of the Liver. Amsterdam, July 2013.

[29] Gidding H F, Topp L, Middleton M, Robinson K, Hellard M, McCaughan G et al. The epidemiology of hepatitis $\mathrm{C}$ in Australia: notifications, treatment uptake and liver transplantations, 1997-2006. J Gastroenterol Hepatol 2009; 24:1648-1654.

[30] Volk M L, Tocco R, Saini S, Lok AS. Public health impact of antiviral therapy for hepatitis C in the United States. Hepatology 2009; 50:1750-1755.

[31] Dore G J. The changing therapeutic landscape for hepatitis C. Med J Aust 2012; 196:629632.

[32] Ghany M G, Liang TJ. Current and future therapies for hepatitis C virus infection. N Engl J Med 2013; 369:679-680.

[33] Delarocque-Astagneau E, Meffre C, Dubois F, Pioche C, Le Strat Y, Roudot-Thoraval F et al. The impact of the prevention programme of hepatitis $C$ over more than a decade: the French experience. J Viral Hepat 2010; 17:435-443.

[34] Bruggmann P, Berg T, Ovrehus AL, Moreno C, Brandao Mello CE, Roudot-Thoraval F et 
al. Historical epidemiology of hepatitis C virus (HCV) in selected countries. J Viral Hepat 2014; 21 Suppl 1:5-33.

[35] Hatzakis A, Wait S, Bruix J, Buti M, Carballo M, Cavaleri M et al. The state of hepatitis B and C in Europe: report from the hepatitis B and C summit conference*. J Viral Hepat 2011; 18 Suppl 1:1-16. 
Table 1. Characteristics of the individuals in the study population, by data collection period of pegI\&R treatment initiation.

\begin{tabular}{|c|c|c|c|c|c|c|c|c|c|c|c|c|}
\hline \multirow[b]{2}{*}{ All patients } & \multicolumn{2}{|c|}{$\begin{array}{l}2001-2 \\
N(\operatorname{col} \%)\end{array}$} & \multicolumn{2}{|c|}{$\begin{array}{l}2003-4 \\
N(\operatorname{col} \%)\end{array}$} & \multicolumn{2}{|c|}{$\begin{array}{l}2005-6 \\
N(\operatorname{col} \%)\end{array}$} & \multicolumn{2}{|c|}{$\begin{array}{l}2007-8 \\
N(\operatorname{col} \%)\end{array}$} & \multicolumn{2}{|c|}{$\begin{array}{l}2009-10 \\
N(\operatorname{col} \%)\end{array}$} & \multicolumn{2}{|c|}{$\begin{array}{l}\text { All years } \\
N(\operatorname{col} \%)\end{array}$} \\
\hline & 237 & - & 496 & - & 696 & - & 906 & - & 1560 & - & 3895 & - \\
\hline $\begin{array}{l}\text { Mean } N \text { per } \\
\text { clinic [range] }\end{array}$ & 18 & [2-55] & \multicolumn{2}{|c|}{$38[10-96]$} & \multicolumn{2}{|c|}{54 [7-138] } & \multicolumn{2}{|c|}{70 [15-139] } & \multicolumn{2}{|c|}{120 [9-258] } & \multicolumn{2}{|c|}{235 [8-564] } \\
\hline Male & 179 & $(76)$ & 351 & (71) & 491 & (71) & 633 & (70) & 1163 & (75) & 2817 & (72) \\
\hline M age (SD) & 43 & (9.3) & 42 & (9.4) & 41 & $(9.4)$ & 42 & (9.6) & 41 & (9.5) & 41 & (9.5) \\
\hline $\begin{array}{c}\text { Diagnosed } \\
\text { cirrhosis }\end{array}$ & 72 & $(30)$ & 119 & (24) & 119 & (17) & 124 & (14) & 164 & (11) & 598 & (15) \\
\hline Genotype 2/3 & 114 & $(48)$ & 280 & (57) & 446 & (64) & 511 & (56) & 889 & (57) & 2240 & (58) \\
\hline Alcohol $>50$ & 43 & (18) & 107 & (22) & 159 & (23) & 202 & (22) & 317 & (20) & 828 & (21) \\
\hline \multicolumn{13}{|l|}{ Risk group } \\
\hline Non-PWID & 100 & $(42)$ & 168 & (34) & 230 & (33) & 259 & (29) & 328 & (21) & 1085 & (28) \\
\hline PWID & 112 & $(47)$ & 274 & (55) & 399 & (57) & 533 & (59) & 1023 & (66) & 2341 & (60) \\
\hline Not known & 25 & $(11)$ & 54 & (11) & 67 & (10) & 114 & (13) & 209 & (13) & 469 & (12) \\
\hline $\begin{array}{l}\text { Prison } \\
\text { referral }\end{array}$ & 3 & (1) & 14 & (3) & 37 & (5) & 43 & (5) & 117 & (8) & 214 & (5) \\
\hline \multicolumn{13}{|l|}{ SVR } \\
\hline All patients & 104 & $(44)$ & 256 & (52) & 415 & (60) & 491 & (54) & 882 & (57) & 2148 & (55) \\
\hline Excl. cirrhotic & ic 84 & $(51)$ & 224 & (59) & 384 & (67) & 464 & (59) & 823 & (59) & 1979 & (60) \\
\hline
\end{tabular}

Note: $\mathrm{M}=$ mean; $\mathrm{N}=$ number of patients; col \% = column percentage; $\mathrm{SD}=$ standard deviation; PWID $=$ people who inject drugs; Alcohol $>50=$ maximum sustained alcohol use greater than 50 units/week. Excl. cirrhotic $=$ study population excluding patients who had been diagnosed with cirrhosis. 
Table 2. Odds-ratio transformed coefficients from the final fitted mixed-effects (adjusted) regression model; the dependent variable is logit-transformed proportion SVR at the clinic level (analysis (c)). Unadjusted ORs are also shown.

\begin{tabular}{llllll} 
& \multicolumn{2}{c}{ Unadjusted } & \multicolumn{2}{c}{ Adjusted } \\
Variable & OR & $\mathbf{( 9 5 \% ~ C I )}$ & OR & $\mathbf{( 9 5 \% ~ C I )}$ & \\
\hline Intercept & - & - & 0.024 & $(0.002-0.323)$ & - \\
Age (per year) & 0.971 & $(0.925-1.019)$ & 1.031 & $(0.980-1.085)$ & \\
Diagnosed cirrhosis & 0.997 & $(0.987-1.008)$ & 1.005 & $(0.996-1.017)$ & \\
Genotype 2 or 3 & 1.024 & $(1.014-1.034)$ & 1.027 & $(1.016-1.037)$ & \\
PWID & 1.009 & $(1.001-1.018)$ & 1.012 & $(1.004-1.021)$ & \\
Prison referral & 1.020 & $(1.000-1.040)$ & 1.010 & $(0.992-1.029)$
\end{tabular}

Note: 95\% CI = approximate 95\% confidence interval; confidence interval; PWID = people who inject drugs 


\section{FIGURE LEGENDS}

Fig. 1. Cumulative number of pegylated interferon \& ribavirin treatment initiations (filled circles) and SVRs (squares) in Scottish specialist hepatitis clinics by calendar year of treatment initiation (analysis (a)). The cumulative number of non-SVRs is the difference between green and red series.

Fig. 2. Spaghetti plots of six covariates over the study period (2001/2002 to 2010), analysis (a). Individual panels show mean age, percent diagnosed cirrhosis, percent maximum sustained alcohol consumption $\geq 50$ units/week, percent genotype $2 / 3$, percent PWID, and percent prison referral. Individual clinics are indicated with grey lines; the across-clinic mean is indicated with a weighted black line.

Fig. 3. SVR over the study period (2001/2 to 2010). Panel A shows SVR stratified by genotype category. Panel B is a spaghetti plot of SVR over the study period, analysis (b), where individual clinics are indicated with grey lines and the across-clinic mean is indicated with a weighted black line. 


\section{APPENDIX}

Table A1. Odds-ratio transformed coefficients from unadjusted and adjusted mixed-effects logistic regression analysis of individual-level data; the dependent variable is SVR.

\begin{tabular}{lcllll} 
& \multicolumn{2}{c}{ Unadjusted } & \multicolumn{3}{c}{ Adjusted } \\
Variable & OR & $(\mathbf{9 5 \%} \mathbf{C I})$ & OR & $\mathbf{( 9 5 \% \mathbf { C I } )}$ \\
\hline Intercept & - & & 2.39 & $(1.56-3.65)$ \\
Age (per year) & 0.97 & $(0.96-0.97)$ & 0.98 & $(0.97-0.98)$ \\
Diagnosed cirrhosis & 0.24 & $(0.20-0.29)$ & 0.26 & $(0.21-0.32)$ \\
Genotype 2 or 3 & 2.74 & $(2.40-3.12)$ & 2.77 & $(2.41-3.17)$ \\
Risk group Non-PWID & Ref. & & Ref. & \\
\multicolumn{2}{r}{$\quad$ PWID } & 0.94 & $(0.81-1.09)$ & 0.82 & $(0.70-0.96)$ \\
\multicolumn{2}{c}{ Not known } & 1.01 & $(0.81-1.27)$ & 0.92 & $(0.73-1.17)$ \\
Prison referral & 0.96 & $(0.96-1.30)$ & 0.89 & $(0.64-1.23)$
\end{tabular}

Note: $\mathrm{OR}=$ odds ratio; $95 \% \mathrm{CI}$ = approximate $95 \%$ confidence interval; PWID = people who inject drugs 
Fig. A1. SVR over the study period (2001/2 to 2010), stratified by genotype category and risk group. Genotype 2/3 is indicated with a solid line; other genotypes with a broken line.

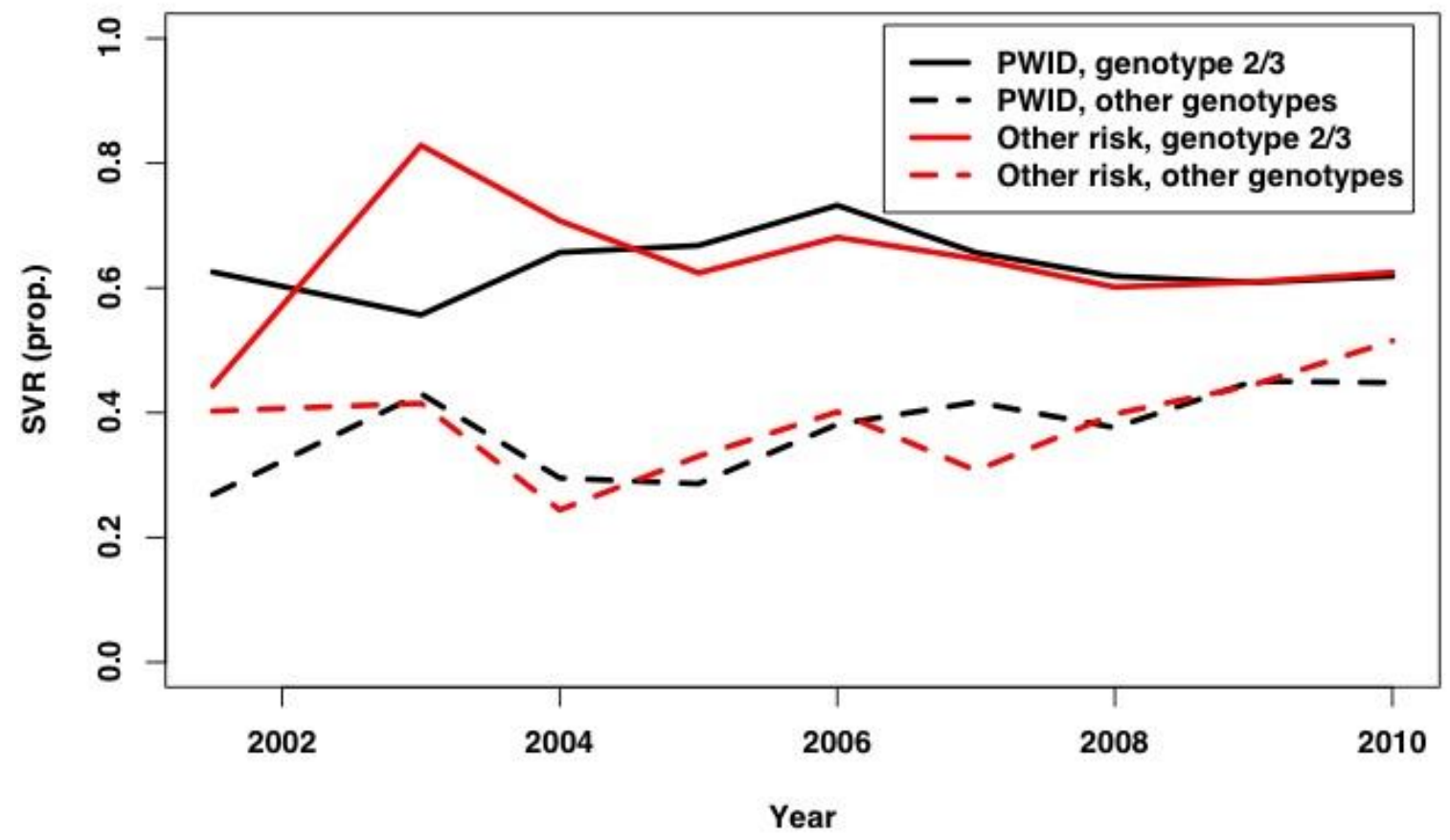


Table A2. Odds-ratio transformed coefficients from fitted mixed-effects regression models applied to the study population without diagnosed cirrhosis $(n=3297)$; the dependent variable is logit-transformed proportion SVR at the clinic level.

\begin{tabular}{llllll} 
& \multicolumn{2}{c}{ Unadjusted } & \multicolumn{2}{c}{ Adjusted } \\
Variable & OR & $\mathbf{( 9 5 \% ~ C I )}$ & OR & $\mathbf{( 9 5 \% ~ C I ) ~}$ & \\
\hline Intercept & - & - & 0.099 & $(0.005-1.850)$ & - \\
Age (per year) & 0.988 & $(0.938-1.041)$ & 1.011 & $(0.954-1.071)$ & \\
Genotype 2 or 3 & 1.021 & $(1.019-1.032)$ & 1.032 & $(1.019-1.044)$ & \\
PWID & 1.008 & $(0.998-1.019)$ & 1.006 & $(0.997-1.017)$ & \\
Prison referral & 1.001 & $(0.977-1.026)$ & 0.995 & $(0.975-1.015)$
\end{tabular}

Note: 95\% CI = approximate 95\% confidence interval; confidence interval; PWID = people who inject drugs 
Fig. A2. Spaghetti plot of SVR over the study period (2001/2 to 2010) for the study population without diagnosed cirrhosis. Individual clinics are indicated with grey lines; the across-clinic mean is indicated with a weighted black line.

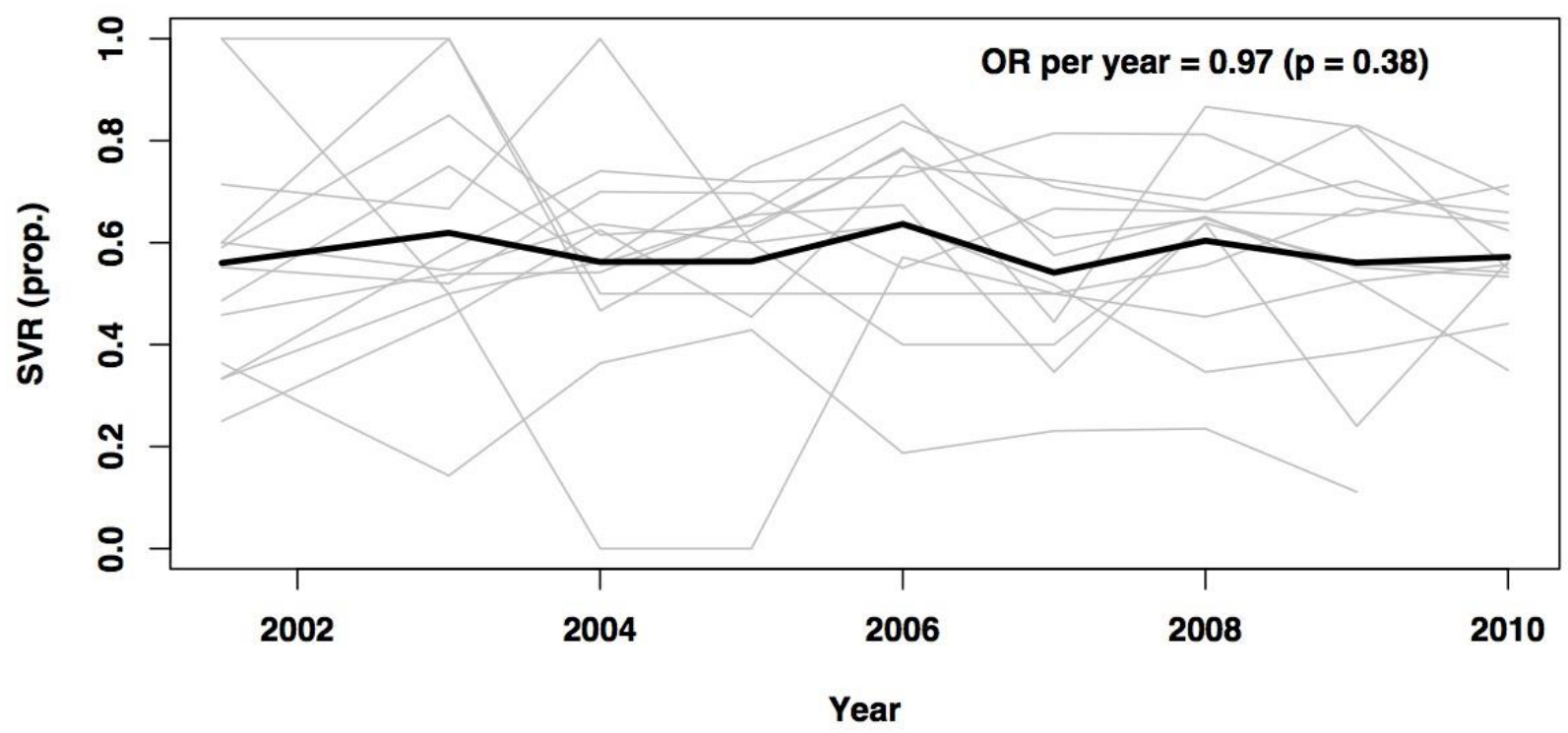

